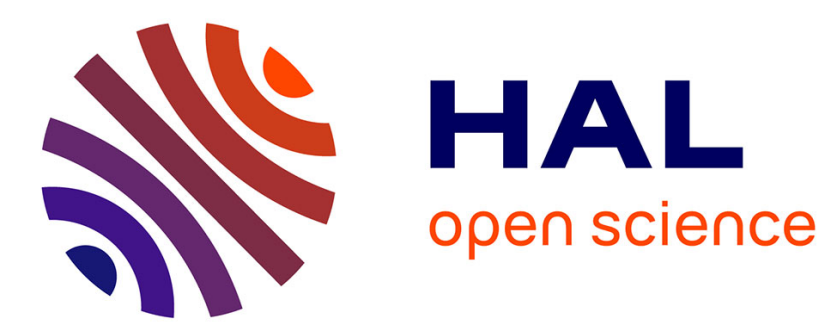

\title{
Transverse field effect close to the critical point in the TGS ferroelectric
}

\author{
Boguslaw Fugiel, Toshio Kikuta, Katarzyna Wojtkow
}

\section{To cite this version:}

Boguslaw Fugiel, Toshio Kikuta, Katarzyna Wojtkow. Transverse field effect close to the critical point in the TGS ferroelectric. Philosophical Magazine, 2011, pp.1. 10.1080/14786435.2011.594815 . hal-00717920

\section{HAL Id: hal-00717920 \\ https://hal.science/hal-00717920}

Submitted on 14 Jul 2012

HAL is a multi-disciplinary open access archive for the deposit and dissemination of scientific research documents, whether they are published or not. The documents may come from teaching and research institutions in France or abroad, or from public or private research centers.
L'archive ouverte pluridisciplinaire HAL, est destinée au dépôt et à la diffusion de documents scientifiques de niveau recherche, publiés ou non, émanant des établissements d'enseignement et de recherche français ou étrangers, des laboratoires publics ou privés. 


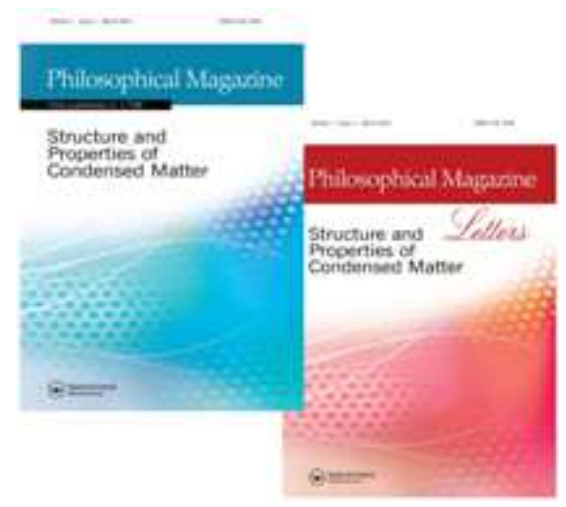

\section{Transverse field effect close to the critical point in the TGS ferroelectric}

\begin{tabular}{|c|c|}
\hline Journal: & Philosophical Magazine \& Philosophical Magazine Letters \\
\hline Manuscript ID: & TPHM-11-May-0168 \\
\hline Journal Selection: & Philosophical Magazine \\
\hline $\begin{array}{r}\text { Date Submitted by the } \\
\text { Author: }\end{array}$ & 04-May-2011 \\
\hline Complete List of Authors: & $\begin{array}{l}\text { Fugiel, Boguslaw; University of Silesia, Institute of Physics } \\
\text { Kikuta, Toshio; University of Toyama } \\
\text { Wojtkow, Katarzyna; University of Silesia }\end{array}$ \\
\hline Keywords: & ferroelectrics, phase transitions, polarization \\
\hline Keywords (user supplied): & TGS, transverse electric field, hysteresis loop \\
\hline
\end{tabular}

\section{SCHOLARONE \\ Manuscripts}




\title{
Transverse field effect close to the critical point in the TGS ferroelectric
}

\author{
Bogusław Fugiel $^{(1)}$, Toshio Kikuta ${ }^{(2)}$ and Katarzyna Wojtków ${ }^{(1)}$ \\ (1) August Chetkowski Institute of Physics \\ University of Silesia, Uniwersytecka 4, 40-007 Katowice, Poland \\ ${ }^{(2)}$ Graduate School of Science and Engineering, \\ University of Toyama, Toyama 930-8555, Japan
}




\title{
Transverse field effect close to the critical point in the TGS ferroelectric
}

\begin{abstract}
A hysteresis loop was measured in a round plate sample of the triglycine sulphate (TGS) ferroelectric with two measurement and one side electrode. Due to a non-zero electric potential $\mathrm{V}_{\mathrm{s}}$ applied to the side electrode the hysteresis loop gradually decayed on time. It has been shown that the higher the $V_{s}$ value, the shorter time $t_{d}$ is required for the hysteresis loop to disappear. The value of $t_{d}{ }^{-1}$ has turned out to be proportional to the electric potential $\mathrm{V}_{\mathrm{s}}$ generating a transverse field at a constant temperature. Within the limits of experimental errors, the inverse of the slope of the dependence $t_{d}{ }^{-1}$ versus $V_{s}$ is proportional to the difference $T_{C}-T$. A relation between the temperature $T$, the spontaneous polarization $\mathrm{P}_{ \pm}$(positive or negative) and the freezing parameter $\mathrm{f}$ has been proposed. The parameter $f$ describes the influence of the transverse electric field. Arguments in favour of considering the transverse field effect as occurring due to free electric charges flowing into the crystal have been given.

A method by means of which the parameters of the hysteresis loop can be easily adjusted by an electric potential of an additional side electrode has been proposed.
\end{abstract}

Keywords: ferroelectrics, TGS, transverse electric field, hysteresis loop

\section{Introduction}

Until recently the results of investigations of uniaxial ferroelectrics placed in an electric field $E_{\perp}$ perpendicular to the polar axis had been reported very rarely. As far as we know, the first experiments concerning electric susceptibility of the ferroelectric triglycine sulphate (TGS) monoclinic crystal exposed to the field $\mathrm{E}_{\perp}$ were carried out by Michailov, Stankowska and Jasiński [1,2]. A few years earlier the transverse electric field was applied during the electric conductivity measurements along different directions [3]. Also magnetic systems placed in a transverse magnetic field were investigated experimentally [4]. Theoretical works concerning both ferroelectric $[5,6]$ and magnetic systems [7-11] in the transverse field were published, too. On the other hand, the influence of the space electric charge and charged defects on the shape of the hysteresis loop was investigated theoretically in bulk ferroelectrics and films [12-14]. There is every indication that an injection of free electric charges into the crystal is particularly efficient if an electric field is directed not parallel to the 
ferroelectric axis. In the present paper we give experimental evidence for the role electric carriers play for changes in physical properties due to the transverse field application.

The existence of a hysteresis loop is the main and inherent feature of ferroelectric materials. Due to the possibility of practical application of ferroelectrics its parameters were intensively investigated. For example, research intended to define an influence of measuring field amplitude and frequency, temperature and pressure, a constant electric field, admixtures and defects, $\mathrm{X}$ and $\gamma$ radiations as well as pale irradiation on the shape and position of the hysteresis loop were carried out [15-21]. In the present paper we propose a very simple method of hysteresis loop modification by means of the electric potential of an additional side electrode. The parameters of the loop can be easily adjusted by this potential.

In order to observe the hysteresis loop on an oscilloscope by means of the Sawyer-Tower method [22], an alternating (measuring) electric field, the so-called driving electric field, is needed. If an additional constant (in time) electric field parallel to the ferroelectric axis is applied, the hysteresis loop is shifted in the horizontal direction. This is a typical reaction to the bias field parallel to the ferroelectric axis. On the other hand, there arises a question: what will happen if a constant electric field is applied perpendicularly to the ferroelectric axis? As appeared $[23,24]$, no instantaneous reaction can be then expected. However, if a prolonged transverse field is applied, in the course of time the hysteresis loop is becoming more and more suppressed and finally disappears.

Such a decay in time for TGS, triglycine selenate (TGSe) and Rochelle salt ferroelectrics was presented for different electrode systems in our former papers [2427]. A series of hysteresis loop decays for TGS is shown in Fig.1. In the measurement 
of Fig.1, a simple Sawyer-Tower circuit was used and no compensation for electric conductivity of the sample was applied. The shape of the sample was a hexahedron and two pairs of gold electrodes were attached to it (see detail in Ref. 24). A spontaneous polarization $\mathrm{P}_{\mathrm{s}}$ is defined by the polarization under no electric field, with no factor that reduces the polarization, such as the back-switching effect. The value of $\mathrm{P}_{\mathrm{s}}$ can be estimated to be almost the same as that of the remanent polarization in the measurement of Fig.1 because the remanent polarization did not vary under various measuring fields with the strength over 3 times greater than that of the coercive field and there was no apparent decrease in the saturation polarization.

Electric conductivity of the sample makes a hysteresis loop inflate and residual capacitance which does not contribute to ferroelectricity makes a hysteresis loop incline [28, 29]. The experimental results shown in Fig.1 suggests that effects mentioned above do not influence the hysteresis loop in any significant way during the application of the transverse electric field $\mathrm{E}_{\perp}$. In Fig.1, during the first stage of the $\mathrm{E}_{\perp}$ field application, a drastic suppression of the remanent polarization is not accompanied by any decrease in the coercive field value. This point differs from the case of the ferroelectric material including charged impurities, like a space charge, where the coercive field value decreases $[12,13]$. Very small coercive field changes due to the space charge could be neglected in the paper [14], though. However, no polarization disappearance was shown there.

Meanwhile, no hysteresis loop disappearance was observed in the longitudinal electric field so far (after a few hundred hours). A difference between the longitudinal and transverse field action has been shown in Fig.2. On the left hand side, one can see an instantaneous shift of the hysteresis loop in a horizontal direction due to an application of the external constant electric field parallel to the ferroelectric axis (by 
means of a blocking capacitor) during the Sawyer-Tower type measurement. On the right hand side, a disappearance of the hysteresis loop is shown as a result of the prolonged external constant transverse electric field application. The lack of any loop corresponds to a stationary state of the crystal in the transverse electric field. Both imprint and fatigue effects are negligible in Fig.2. In the case of the longitudinal field in Fig.2 the effect is not permanent, i.e. the shift disappears after the constant field disconnection. On the other hand, the lack of the hysteresis loop can be still observed at a constant temperature after the transverse field switching off provided that the 'rejuvenating' driving field is not applied for too long [23]. The original properties of the crystal can be easily restored by annealing the sample (rejuvenation) above the critical temperature. This method seems to be the most efficient way of rejuvenation.

In the case of the very popular TGS ferroelectric, a time $t_{d}$ of about $10^{1}-10^{2}$ hours is usually required for the loop to disappear in a transverse electric field of the order of $10^{2} \mathrm{kV} / \mathrm{m}$, at room temperature. That is probably the reason why the properties of ferroelectrics with hydrogen bonds, observed after their exposure to an electric field not parallel to the ferroelectric axis, remained unknown for many years. The time $t_{d}$ depends on temperature [24] or, to be more precise, on how far away from the critical point of the paraelectric-ferroelectric phase transition we are (in the ferroelectric phase).

In the monoclinic TGS electric conductivity along the $\mathrm{c}$ axis direction is higher that those along $\mathrm{a}$ and $\mathrm{b}$ axes $[3,30]$. On the other hand, the influence of the transverse field applied along the $\mathrm{c}$ axis is particularly significant $[24,31]$. Therefore, there are arguments in favour of considering the transverse field effect as occurring due to free electric charges flowing into the crystal [24]. For it is just the high concentration of free charges that may cause a freezing of domains [32, 33] and 
therefore a reduction of the hysteresis loop. On the other hand, the appearance of quite a new rigid domain structure $[32,33]$ with domain walls turned by 90 degrees as well as transverse polarization of the crystal which spontaneously increases while lowering temperature in the zero external electric field (transverse quasi-pyroelectric effect) $[25,34]$ suggest that the nature of the effect under consideration is more complex. In particular, it cannot be understood simply as an electret effect. This is not a relaxorlike system, either, because of a low frequency suppression observed experimentally in TGS [35]. This complexity seems to be confirmed also by the fact that, although similar electric conductivity values were obtained in TGS along the a and b axes [3, 30], no disappearance of hysteresis loop has been observed in longitudinal (parallel to the ferroelectric $b$ axis) electric fields so far.

\section{Experimental}

Below in the present paper we show the experimental results obtained on a round TGS sample with two symmetrically attached circular silver paste measurement electrodes and one silver paste side-ring-electrode (Fig.3). The paste Leitsilber 200 Hans Wolbring GmbH was used. The diameter of the sample, the diameter of the measurement electrodes and the sample width were $(7.6 \pm 0.2) \mathrm{mm},(4.7 \pm 0.2) \mathrm{mm}$ and (3.0 \pm 0.1$) \mathrm{mm}$, respectively. The crystal was grown in the Toyama University in Japan. The sample was connected to a typical Sawyer-Tower measurement circuit. The amplitude and the frequency of the measuring voltage (as a source of the driving field) were $2.5 \mathrm{~V}$ and $100 \mathrm{~Hz}$, respectively. This sin-wave voltage was generated by means of the generator Agilent 33120A and amplified by the bipolar operation power supply/amplifier KEPCO BOP 1000M. The constant ground-referenced electric potential $\mathrm{V}_{\mathrm{s}}$ was applied by means of the power supply ZWN-42 POLON to the side 
electrode at a given temperature. At the same time the upper measurement electrode remained grounded, except for short moments when the hysteresis loop was observed. The values of the time $t_{d}$ after which the hysteresis loop disappeared were measured at different temperatures and side potentials.

Just before the hysteresis loop disappearance its shape was carefully observed for a few minutes in order to make sure that the loop was of ferroelectric character. When the remanent polarization was one order of magnitude less than that of the original sample, the coercive field could be observed to decrease (together with further remanent polarization suppression and linearization of the polarization field dependence), which finally led to the loop closing. Slight corrections by means of a variable resistor allowed us to distinguish then between the intrinsic ferroelectric hysteresis loop and the pseudo-loop appearing due to the electric conductivity and the residual capacitance of the sample. The time $t_{d}$ was detected with the accuracy of 1 minute.

The similar sample-electrode system was used in $[27,36]$ but then blocking capacitors had to be connected in order to apply the constant electric potential to the measurement electrodes. The measurement circuit without any blocking capacitors shown in the present paper was used by us for the first time.

\section{Results}

In Fig.4 we can see that the inverse of $t_{d}$ is a linear function of $V_{s}$, at $T=319.25 K$. A similar dependence

$$
\frac{1}{t_{d}}=\text { slope } V_{s}
$$

was also observed at other temperatures, though with different slopes. In other words, the inverse of the slope is a well-defined parameter for a given temperature. In insert 
of Fig.4 one can see a temperature dependence of the inverse of the slope which is linear within the limits of experimental errors. The point with $\operatorname{slope}^{-1}=0$ at the critical temperature $\mathrm{T}_{\mathrm{C}}=322.1 \mathrm{~K}$ was added as being ex definitione equal to zero. The value of $\mathrm{T}_{\mathrm{C}}=(322.1 \pm 0.1) \mathrm{K}$ was taken as the temperature of the hysteresis loop disappearance (or appearance) in the rejuvenated TGS sample. There is no theoretical prediction concerning the slope ${ }^{-1}$ versus temperature dependence in the neighbourhood of the critical point. Therefore, we have chosen the simplest, i.e. the linear fit to the experimental points. We can conclude that the transverse field effect has a critical character because the slope tends to infinity when the temperature approaches the critical point.

\section{Discussion}

\subsection{Freezing parameter $f_{0}$}

A concept of freezing parameter $\mathrm{f}$ has been introduced in Ref.36. Although the physical meaning of $\mathrm{f}$ is still being investigated, it is a convenient parameter for describing the influence of the transverse field. For a sample never influenced by a transverse electric field or for the crystal rejuvenated by annealing above $T_{C}$ the value $\mathrm{f}=0$ is postulated. When the transverse field is being applied, however, the hysteresis becomes more and more suppressed and finally disappears. At the same time f rises gradually from zero to a border value $\mathrm{f}_{0}$. The spontaneous polarization is equal to zero for $f \geq f_{0}$. The border value $f_{0}$ depends on temperature.

As becomes evident from Fig.4, the higher the $\mathrm{V}_{\mathrm{s}}$ potential, the shorter period of time is required for the hysteresis loop to disappear. This suggests that the influence of the transverse field on the hysteresis loop shape can be viewed as resulting from electric carriers flowing into the crystal [24]. At a given temperature 
there exists a minimal value $\mathrm{n}=\mathrm{n}_{0}$ of concentration of free electric carriers (corresponding to the border value $\mathrm{f}=\mathrm{f}_{0}$ ) which is required for the hysteresis to disappear. In fact, as follows from Fig.4, the value of the product $V_{s} t_{d}=$ slope $^{-1}$ determines the conditions for the hysteresis loop disappearance. This suggests an interpretation of the parameter $f_{0}$ which tends to zero when the critical point is approached. We propose the relation

$$
f_{0}=\operatorname{slope}^{-1}
$$

For temperatures close to $T_{C}$ the freezing parameter $f_{0}$ can be viewed as proportional to the electric charge $\mathrm{q}_{0}$ flowing through the crystal or being injected into the crystal during the time $t_{d}$. In fact, we have

$$
q_{0} \sim \int_{0}^{t_{d}} V_{s} R^{-1} d t
$$

where $\mathrm{R}$ is the resistance of the sample. If we assume that $\mathrm{R}$ does not depend on time, the following relation

$$
f_{0} \sim q_{0}
$$

is obtained, where $\mathrm{R} \approx$ const. within a narrow temperature interval below $\mathrm{T}_{\mathrm{C}} . \mathrm{A}$ possibility of generalizing this relation for a broader temperature region is discussed in one of the next paragraphs.

\subsection{Relation between $P, T$ and $f$}

A linear temperature dependence of $\mathrm{f}_{0}$ results from the insert of Fig.4 (for zero spontaneous polarization and $\mathrm{f}=\mathrm{f}_{0}=$ slope $^{-1}$ ). On the other hand, the Landau type of the spontaneous polarization temperature dependence can be assumed for $f=0$, i.e. for the original or rejuvenated sample. Consequently, for $T<T_{C}$ we can consider the empirical equation, representing also the case $0<\mathrm{f}<\mathrm{f}_{0}$ and $\mathrm{P}_{ \pm} \neq 0$ : 


$$
P_{ \pm}= \pm B\left(T_{c}-T\right)^{1 / 2} \mp F f^{1 / 2}
$$

where $\mathrm{P}_{+}>0$ and $\mathrm{P}_{-}=-\mathrm{P}_{+}$are positive and negative spontaneous polarizations (parallel to the ferroelectric axis), respectively, $\mathrm{T}_{\mathrm{C}}$ is the critical temperature of the paraelectric-ferroelectric phase transition, $\mathrm{B}>0$ and $\mathrm{F}>0$ are constants. Because of an almost rectangular hysteresis loop observed for TGS, the spontaneous polarization can be approximated by the remanent one. The formula (5) should be considered valid only within the limited $T_{C}-T$ and $f_{0}-f$ value region, as it is for example in the case of critical exponents. As results from Eq.5, the difference $\mathrm{P}_{ \pm}\left(\mathrm{T}, \mathrm{f}_{2}\right)-\mathrm{P}_{ \pm}\left(T, \mathrm{f}_{1}\right)$ for $\mathrm{f}_{2} \neq \mathrm{f}_{1}$ does not depend on temperature. This is in agreement with the experimental data (for relatively small spontaneous polarization values, i.e. for small difference $f_{0}-f>0$ ) shown in Fig.5 of Ref.37. For f=0, Eq.5 gives the Landau type relation:

$$
P_{ \pm}=P_{0 \pm}= \pm B\left(T_{c}-T\right)^{1 / 2}
$$

as in the case of a sample never influenced by a transverse field. On the other hand, during a transverse electric field application at a constant temperature, the value of $\mathrm{f}$ increases from zero to $\mathrm{f}_{0}$. At the same time the spontaneous polarization decreases, according to Eq.5. Finally, for $\mathrm{f}=\mathrm{f}_{0}$ we have $\mathrm{P}_{ \pm}=0$. As results from Eq.5, the border value $\mathrm{f}=\mathrm{f}_{0}$ decreases linearly with temperature

$$
f_{0}(T)=\left(B F^{-1}\right)^{2}\left(T_{c}-T\right)
$$

This is in accordance with the data in insert of Fig. 4 and the definition (2). In this case we have $f_{0} \sim P_{0 \pm}{ }^{2}$, i.e. a critical exponent $2 \beta$ is expected for $f_{0}$ as a function of temperature.

On the basis of the relation (5) we can draw the functions $P_{ \pm}(f, T), f(T)$ and $\mathrm{P}_{ \pm}(\mathrm{f})$ much more precisely (e.g. with different convexity) than that shown schematically in Ref.36 for TGSe. The function $\mathrm{P}_{+}=\mathrm{P}_{+}(\mathrm{T}, \mathrm{f})$ which results from Eq.5 has been presented in Fig.5. The white dotted line represents the spontaneous 
polarization-temperature dependence for a constant $f>0$. One can see that the lefthand partial derivative $\partial \mathrm{P}_{ \pm} / \partial \mathrm{T}$ at the temperature of the remanent polarization disappearance is then finite. This is in agreement with the experimental results shown in Refs. 36 and 37. We can conclude that only for $f=0$ the tangent to the curves $\mathrm{P}_{ \pm}(\mathrm{T})$ at $\mathrm{T}=\mathrm{T}_{\mathrm{C}}$ is vertical whereas for $\mathrm{f}>0$ it is oblique at temperatures $\mathrm{T}<\mathrm{T}_{\mathrm{c}}$ of the reduced remanent polarization disappearance.

\subsection{Freezing parameter in a broader temperature region}

The results of the present paper indicate that the transverse field effect is strongly correlated with the paraelectric-ferroelectric phase transition. On the other hand, our present data do not seem to be in contradiction to the former ones [24], where the Arrhenius-type dependence of a characteristic time

$$
\tau=\tau_{0} \exp \left(\frac{U_{a}}{k T}\right)
$$

was proposed for the sample of the form of a perpendicular parallelepiped. The time $\tau_{0}$ decreased with rising transverse field intensity. The activation energy and the Boltzmann constant are denoted by $\mathrm{U}_{\mathrm{a}}$ and $\mathrm{k}$, respectively. Taking into account the relation (6) we can consider a general formula for a temperature interval broader than that in Fig. 4

$$
\operatorname{slope}^{-1}(T)=V_{s} t_{d}=\text { const. }\left(T_{C}-T\right) \exp \left(\frac{U_{a}}{k T}\right)
$$

The factor $\left(\mathrm{T}_{\mathrm{C}}-\mathrm{T}\right)$ reflects the crystal properties manifesting themselves close to the ferroelectric-paraelectric phase transition. It can be thought to be an approximation of a more general function $\mathrm{h}(\mathrm{T})$ (corresponding to $\tau_{0}$ in Eq.6) which does not prevail at lower temperatures. However, for $\mathrm{T}$ close to $\mathrm{T}_{\mathrm{C}}$ it can be approximated by a linear dependence, i.e. $h(T) \sim\left(T_{C}-T\right)$. On the other hand, for $T \approx T_{C}$, the Arrhenius factor should be treated as proportional to a weakly temperature-dependent function 
$\exp (1 / \mathrm{T}) \approx \exp \left(1 / \mathrm{T}_{\mathrm{C}}\right)$, and in the limit $\mathrm{T} \rightarrow \mathrm{T}_{\mathrm{C}}$ (i.e. within a few degrees below $\mathrm{T}_{\mathrm{C}}$, see Fig.6) we obtain the dependence shown in insert of Fig.4.

The concept of the freezing parameter can be extended to a broader temperature region, but where $\mathrm{h}(\mathrm{T}) \sim\left(\mathrm{T}_{\mathrm{C}}-\mathrm{T}\right)$ (cf. Fig.6) and $\mathrm{t}_{\mathrm{d}}{ }^{-1} \sim \mathrm{V}_{\mathrm{s}}$ relations are still observed. Let the Arrhenius exponential factor in (7) represent the temperature dependence of resistance $R \sim \exp \left[U_{a} /(k T)\right]$ of the sample $[3,24,30]$. Then the relation (4) is also valid if we take into account Eq.7 and assume that $\mathrm{R}$ does not depend on time in (3).

\section{Summary}

There is a fundamental difference between the mechanism of longitudinal and transverse polarization of the TGS crystal. During the application of an electric field not parallel to the ferroelectric axis the hysteresis loop gradually decreases and finally disappears. It has been shown, however, that such a field does not have to be either perpendicular to the polar axis or homogeneous. The hysteresis loop disappears also in an inhomogeneous electric field with a non-zero transverse component, as it is in the case of the round plate sample with three electrodes. Then the parameters of the hysteresis loop can be easily adjusted by the electric potential of the side electrode.

The disappearance of the hysteresis loop has been observed in a round plate TGS sample after the sufficiently long time $t_{d}$ of applying the side electric potential $\mathrm{V}_{\mathrm{s}}$. For a given temperature $\mathrm{T}<\mathrm{T}_{\mathrm{C}}$, the relation $\mathrm{t}_{\mathrm{d}}{ }^{-1} \sim \mathrm{V}_{\mathrm{s}}$ is valid. On the other hand, the value of the $t_{d} V_{s}$ product changes linearly with temperature and tends to zero when $\mathrm{T}$ approaches $\mathrm{T}_{\mathrm{C}}$. The transverse field effect has a critical nature because the slope of the linear relation $\mathrm{t}_{\mathrm{d}}{ }^{-1}$ versus $\mathrm{V}_{\mathrm{s}}$ tends then to infinity. The classic temperature dependence $\mathrm{P}_{0 \pm}(\mathrm{T})$ can be treated as a special case of a more general relation (Eq.5) between temperature, spontaneous polarization and the freezing parameter $f$. The 
value of the freezing parameter reflects the concentration of free electric charges in the sample. Experimental results confirming the validity of the formula (5) have been cited.

\section{References}

1. S.R. Stoyanov, M.P. Michailov, J. Stankowska, Acta Phys.Polon. A65 (1984) p.141.

2. J. Stankowska, T. Jasiński, Acta Phys. Polon. A67 (1985) p.1059.

3. B. Hilczer and M. Michalczyk, Ferroelectrics 22 (1978) p.721.

4. H. Suzuki and Z. Watanabe, J.Phys.Soc.Jpn. 30 (1971) p.367.

5. C.L. Wang, C. Arago, J. Garcia and J.A. Gonzalo, Physica A 308 (2002) p.337.

6. I.V. Stasyuk and O.V. Velychko, Ferroelectrics 316 (2005) p. 51.

7. H. Thomas, Phys.Rev. 187 (1969) p. 630.

8. J. Sznajd, Acta Phys.Polon., A40 (1971) p. 687.

9. K. Durczewski, J.Phys. C: Solid State Phys. 6 (1973) L413.

10. J. Sznajd, Phys. Rev. B 64 (2001) 052401.

11. J. Sznajd, Phys. Rev. B 65 (2002) 224429.

12. A.N. Morozovska, Ferroelectrics 317 (2005) p. 229.

13. P. Zubko, D.J. Jung, J.F. Scott, J. Appl.Phys. 100 (2006) 114112.

14. A.M. Bratkovsky, A.P. Levanyuk, Phys.Rev.B 61 (2000) p.15042.

15. N. Nakatani, J. Phys.Soc.Jpn. 32 (1972) p.1556.

16. J. Janta, J.Phys.Soc.Jpn. 28, Suppl. (1970) p. 340

17. J. Stankowski, A. Gałęzewski, S. Waplak, U.Gruszczyńska, H.Gierszal, Ferroelectrics 6 (1974) p.209

18. A.G. Chynoweth, Phys.Rev. 113 (1959) p.159.

19. J. Eisner, Ferroelectrics 8 (1974) p. 621.

20. E. Fatuzzo, Helv.Phys.Acta 33 (1960) p.501.

21. E. Fatuzzo, W.J.Merz, Ferroelectricity, North-Holland Publishing Company, Amsterdam, 1967, p.252.

22. C.B. Sawyer and C.H. Tower, Phys.Rev.35 (1930) p.269. 
23. K. Ćwikiel, B. Fugiel and M. Mierzwa, J.Phys.: Condens. Matter 12 (2000) p. 5033.

24. T. Kikuta, H. Nishizuka, T. Yamazaki and N. Nakatani, Ferroelectrics 336 (2006) p.91.

25. B. Fugiel, K. Ćwikiel and W. Serweciński, J. Phys.: Condens. Matter 14 (2002) p.11837.

26. B. Fugiel, Eur. Phys. J. B 61 (2008) p.159.

27. B. Fugiel, J. Phys.: Condens. Matter 20 (2008) 255206.

28. K.Diamant, K.Drenck and R.Pepinsky, Rev.Sci.Instr. 28 (1957) p.30.

29. Chen Xiaogang, Yao Xi, Zhang Desheng and Zhang Liangying, Ferroelectrics 259 (2001) p.55.

30. W. Osak, Z.Naturforsch. 52a (1997) p.621.

31. K. Ćwikiel, B. Fugiel, and M. Mierzwa, J. Phys.: Condens. Matter 11 (1999) p. 7585.

32. K. Ćwikiel, B. Fugiel, and M. Mierzwa, Physica B 293 (2000) p. 58.

33. T. Kikuta, T. Yamazaki and N. Nakatani, J.Korean Phys.Soc. 51 ( 2007) p.754.

34. B. Fugiel, Solid State Commun. 122 (2002) p.237.

35. B. Fugiel, K. Ćwikiel and M. Mierzwa, J.Phys.: Condens. Matter 12 (2000) p.9993.

36. B. Fugiel, J.Phys.: Condens. Matter 20 (2008) 485217.

37. K. Ćwikiel, B. Fugiel and M. Mierzwa, Physica B 296 (2001) p.361. 
Figure captions

Figure 1. Time dependence of the P-E hysteresis loops of TGS under the transverse electric field $\mathrm{E}_{\perp}$ application by a simple Sawyer-Tower circuit. The measurements were carried out at $312.5 \mathrm{~K}$ under the conditions of a triangle-wave driving electric field E parallel to the ferroelectric $b$ axis with the amplitude and frequency of 40 $\mathrm{kV} / \mathrm{m}$ and $5 \mathrm{~Hz}$, respectively, and the transverse DC electric field $\mathrm{E}_{\perp}$ of $200 \mathrm{kV} / \mathrm{m}$ parallel to the $c$ axis.

Figure 2. Hysteresis loops observed on the oscilloscope screen before and after longitudinal $E_{\|}$(instantaneous reaction, not permanent effect) and transverse $E_{\perp}$ (gradual reaction, permanent effect) constant external electric fields E application, drawn schematically. The diagrams concern stationary states of the crystal, i.e. when the loops do not change in time any more; $\mathrm{E}=\left(\mathrm{E}_{\|}^{2}+\mathrm{E}_{\perp}^{2}\right)^{1 / 2}$; both imprint and fatigue effects are negligible here.

Figure 3. Hysteresis loop disappearance drawn schematically, the measurement circuit and the sample with three electrodes; insert: the sample seen from one side with measurement electrodes thicker than in reality (for clarity); $\mathrm{V}_{\mathrm{s}}$ is a ground-referenced electric potential 
Figure 4. Experimental dependence $t_{d}{ }^{-1}$ versus $V_{s}$ at $T=319.25 K$; insert: inverse of slope (in V h) versus temperature.

Figure 5. The 3D relation $\mathrm{P}_{+}=\mathrm{P}_{+}(\mathrm{T}, \mathrm{f})$ drawn on the basis of Eq.(5); a region with no hysteresis loop is shown on one side of the line $f=f_{0}(T)$; the projection onto the spontaneous polarization-temperature plane is shown schematically on the right hand side

Figure 6. Schematic diagrams $\mathrm{C}_{0}(\mathrm{~T})=\left(\mathrm{T}_{\mathrm{C}}-\mathrm{T}\right) \exp \left(\mathrm{A} / \mathrm{T}_{\mathrm{C}}\right)$ and $\mathrm{C}(\mathrm{T})=\left(\mathrm{T}_{\mathrm{C}}-\mathrm{T}\right)$ $\exp (\mathrm{A} / \mathrm{T})$ versus temperature, where $\mathrm{A}$ is a constant; inserts: corresponding Arrhenius type relation 


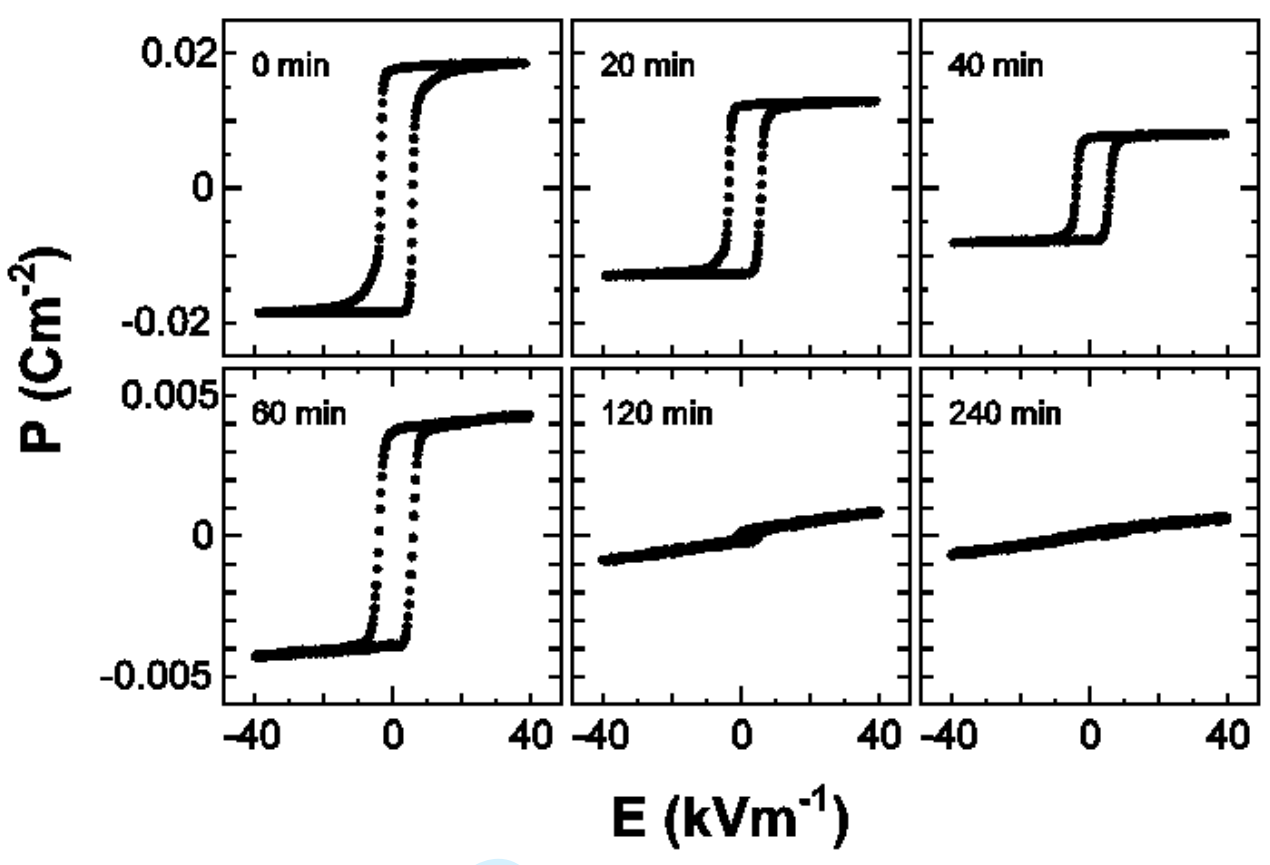

Figure 1. Time dependence of the P-E hysteresis loops of TGS under the transverse electric field $E_{\perp}$ application by a simple Sawyer-Tower circuit. The measurements were carried out at $312.5 \mathrm{~K}$ under the conditions of a triangle-wave driving electric field E parallel to the ferroelectric $b$ axis with the amplitude and frequency of 40 $\mathrm{kV} / \mathrm{m}$ and $5 \mathrm{~Hz}$, respectively, and the transverse DC electric field $\mathrm{E}_{\perp}$ of $200 \mathrm{kV} / \mathrm{m}$ parallel to the $c$ axis. 


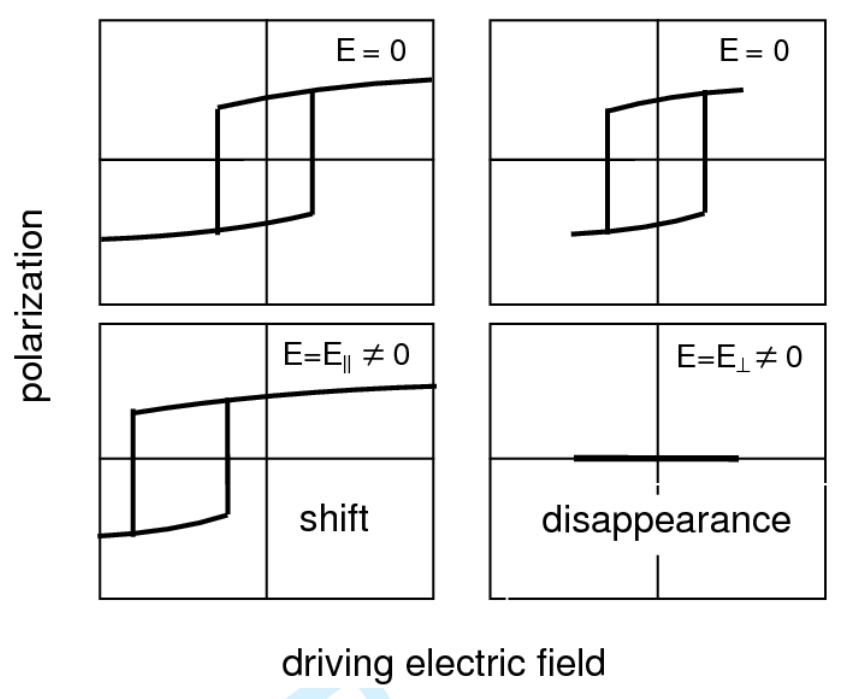

Figure 2. Hysteresis loops observed on the oscilloscope screen before and after longitudinal $\mathrm{E}_{\|}$(instantaneous reaction, not permanent effect) and transverse $\mathrm{E}_{\perp}$ (gradual reaction, permanent effect) constant external electric fields E application, drawn schematically. The diagrams concern stationary states of the crystal, i.e. when the loops do not change in time any more; $\mathrm{E}=\left(\mathrm{E}_{\|}^{2}+\mathrm{E}_{\perp}^{2}\right)^{1 / 2}$; both imprint and fatigue effects are negligible here. 

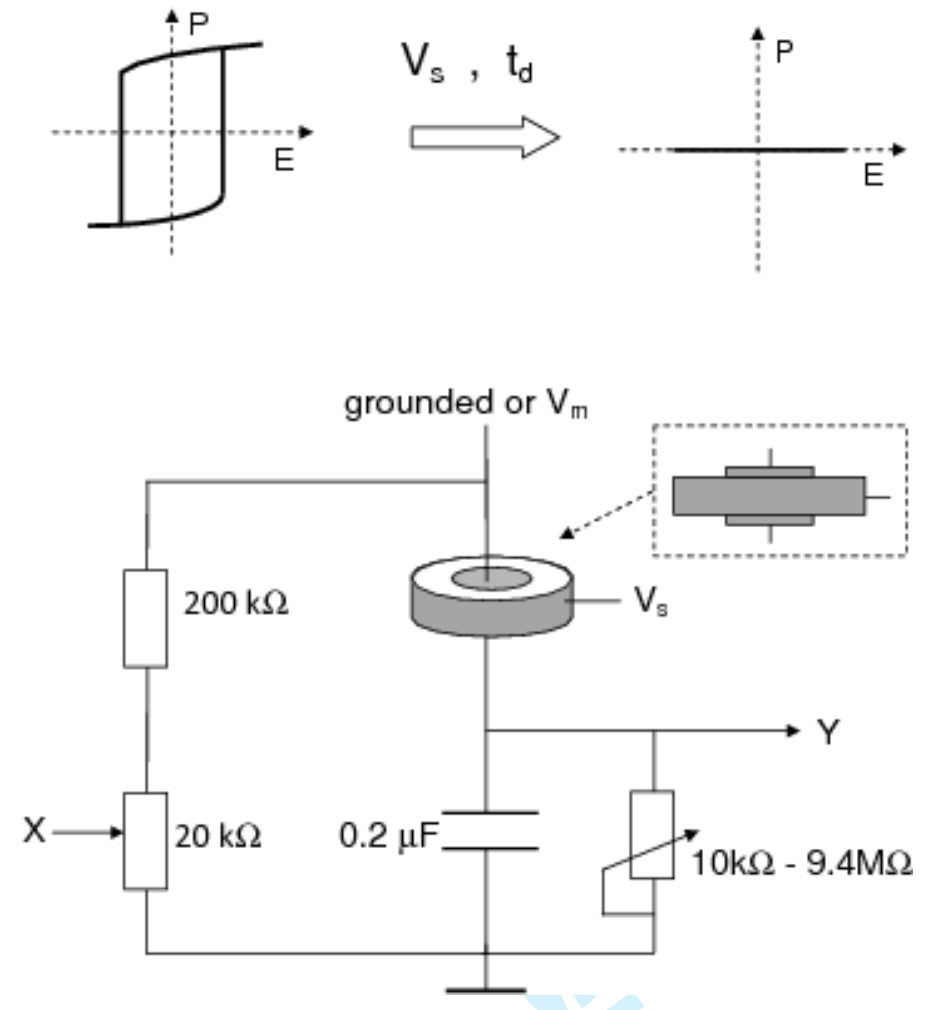

Figure 3. Hysteresis loop disappearance drawn schematically, the measurement circuit and the sample with three electrodes; insert: the sample seen from one side with measurement electrodes thicker than in reality (for clarity); $\mathrm{V}_{\mathrm{s}}$ is a ground-referenced electric potential 


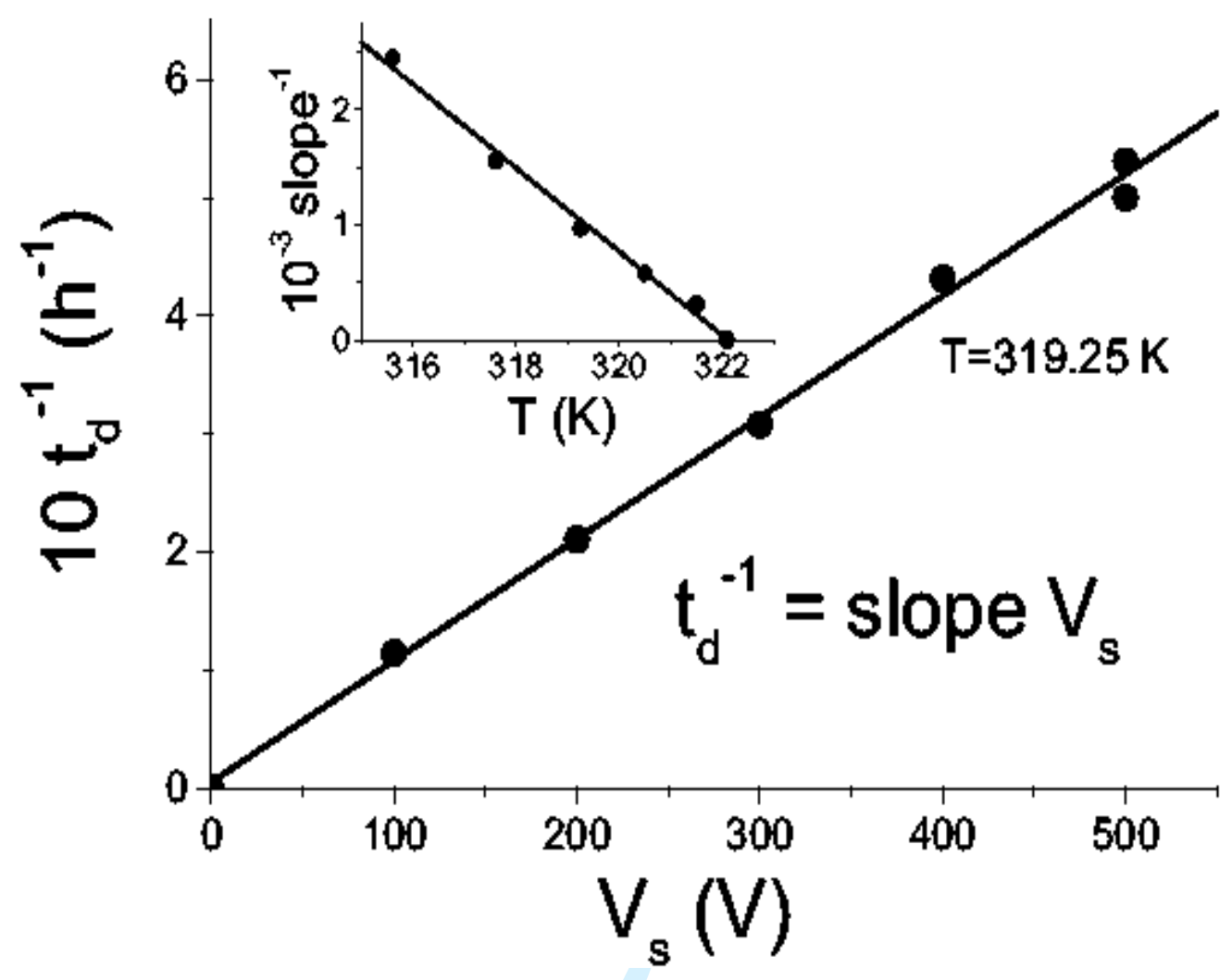

Figure 4. Experimental dependence $\mathrm{t}_{\mathrm{d}}{ }^{-1}$ versus $\mathrm{V}_{\mathrm{s}}$ at $\mathrm{T}=319.25 \mathrm{~K}$; insert: inverse of slope (in V h) versus temperature. 

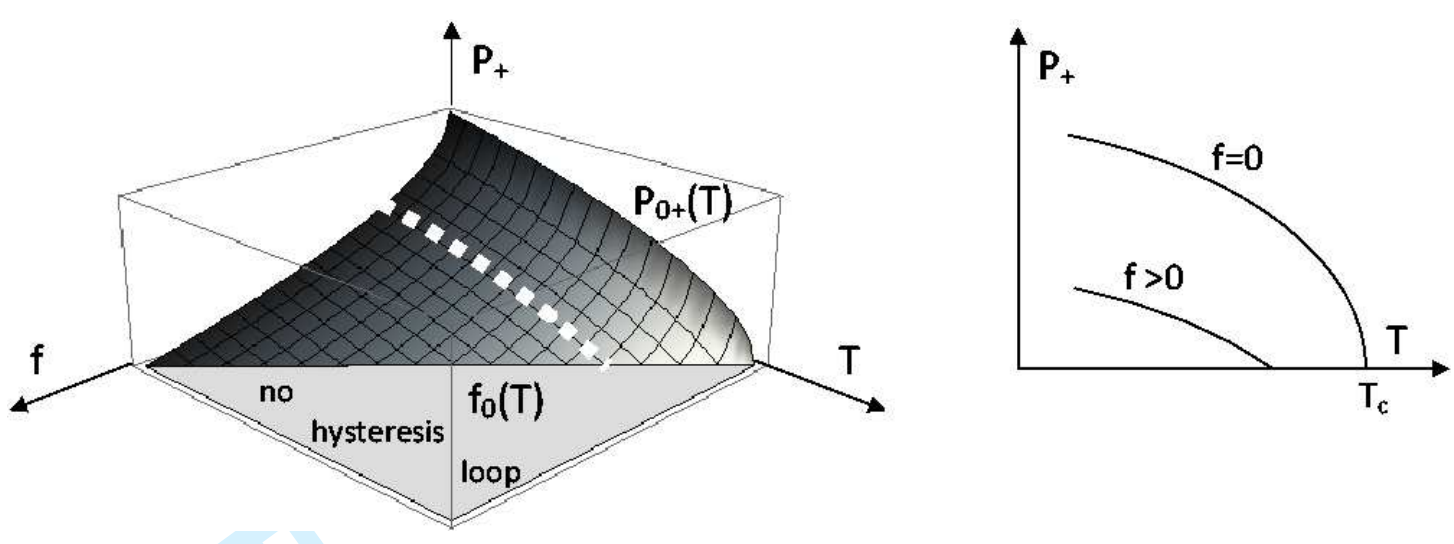

Figure 5. The 3D relation $\mathrm{P}_{+}=\mathrm{P}_{+}(\mathrm{T}, \mathrm{f})$ drawn on the basis of Eq.(5); a region with no hysteresis loop is shown on one side of the line $\mathrm{f}=\mathrm{f}_{0}(\mathrm{~T})$; the projection onto the spontaneous polarization-temperature plane is shown schematically on the right hand side 


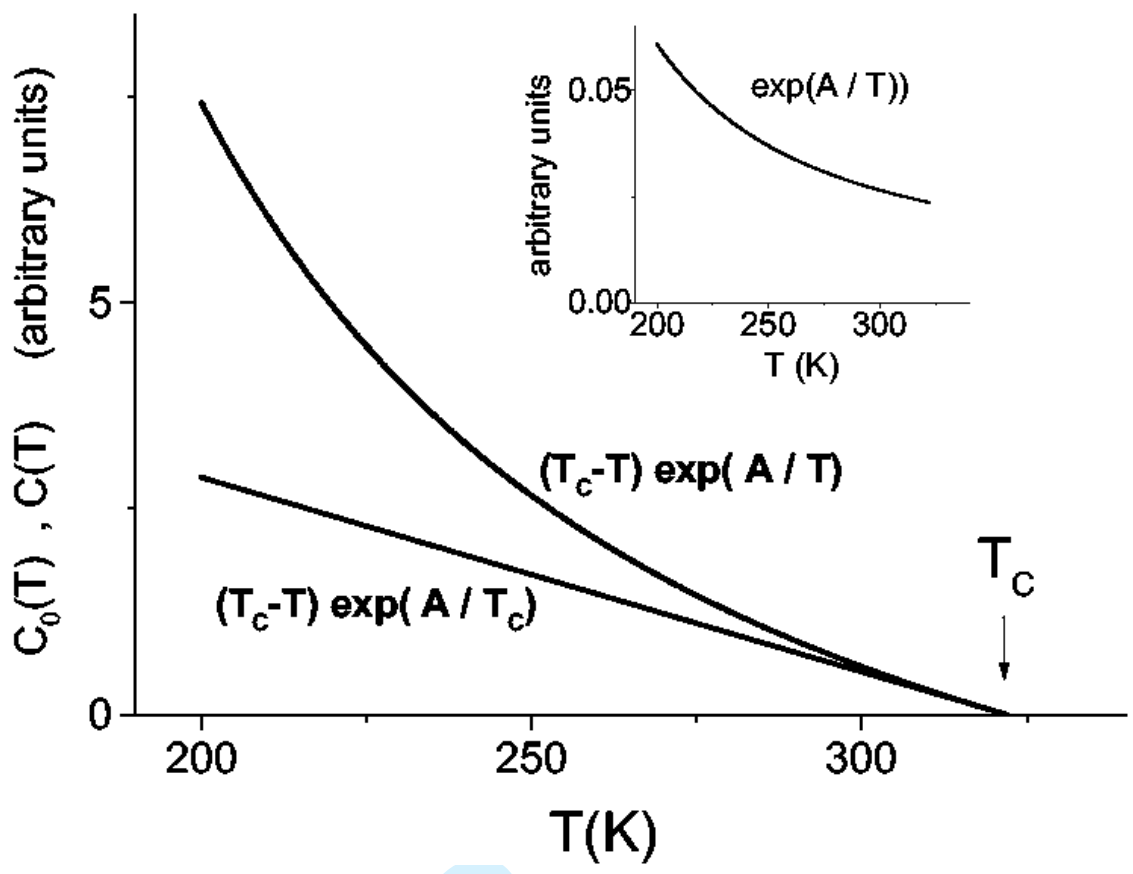

Figure 6. Schematic diagrams $\mathrm{C}_{0}(\mathrm{~T})=\left(\mathrm{T}_{\mathrm{C}}-\mathrm{T}\right) \exp \left(\mathrm{A} / \mathrm{T}_{\mathrm{C}}\right)$ and $\mathrm{C}(\mathrm{T})=\left(\mathrm{T}_{\mathrm{C}}-\mathrm{T}\right)$ $\exp (\mathrm{A} / \mathrm{T})$ versus temperature, where $\mathrm{A}$ is a constant; inserts: corresponding Arrhenius type relation 